\title{
MicroRNA-181a-5p Promotes Osteosarcoma Progression via PTEN/AKT Pathway
}

\author{
Chen Sun, ${ }^{1}$ Chi Chen, ${ }^{1}$ Zhen Chen, ${ }^{2}$ Jun Guo, ${ }^{1}$ Zhi-Hong Yu, ${ }^{1}$ Wei Qian, ${ }^{1}$ Fen Ai ${ }^{1},{ }^{2}$ \\ Liang Xiao $\mathbb{D}^{1},{ }^{1}$ and Xiao Guo ${ }^{1}{ }^{1}$ \\ ${ }^{1}$ Department of Orthopedic Surgery, Renmin Hospital, Hubei University of Medicine, Shiyan, Hubei, China \\ ${ }^{2}$ Department of Emergency, The Central Hospital of Wuhan, Tongji Medical College, Huazhong University of Science \\ and Technology, Wuhan, Hubei, China
}

Correspondence should be addressed to Xiao Guo; gxfragrans@163.com, Liang Xiao; drxl1981@163.com, and Fen Ai; profaf@163.com

Received 26 December 2021; Revised 12 February 2022; Accepted 17 February 2022; Published 8 March 2022

Academic Editor: Makoto Endo

Copyright (c) 2022 Chen Sun et al. This is an open access article distributed under the Creative Commons Attribution License, which permits unrestricted use, distribution, and reproduction in any medium, provided the original work is properly cited.

Osteosarcoma is the most common primary malignant bone tumor in children and adolescents with poor prognosis. MicroRNA181a-5p (miR-181a-5p) is involved in the progression of various tumors; however, its role and underlying mechanism in osteosarcoma remains unclear. In this study, we found that miR-181a-5p was upregulated in human osteosarcoma cells and tissues. miR-181a-5p mimic significantly promoted, while miR-181a-5p inhibitor blocked the proliferation, colony formation, migration, invasion, and cell cycle progression of osteosarcoma cells. Mechanistically, miR-181a-5p bound to the $3^{\prime}$-untranslational region of phosphatase and tensin homolog (PTEN) and reduced its protein expression, thereby activating protein kinase B (PKB/AKT) pathway. Either PTEN overexpression or AKT inhibition notably blocked the tumor-promoting effects of miR-181a-5p. Moreover, we observed that miR-181a-5p mimic further inhibited growth of human osteosarcoma cells in the presence of adriamycin or cisplatin. Overall, miR-181a-5p promotes osteosarcoma progression via PTEN/AKT pathway and it is a promising therapeutic target to treat osteosarcoma.

\section{Introduction}

Osteosarcoma is the most common primary malignant bone tumor in children and adolescents and contributes to $8.9 \%$ of cancer mortality among children [1-3]. Despite the achievements in diagnosis and treatment, the prognosis of osteosarcoma patients remains unsatisfactory with a 5-year overall survival rates no more than $30 \%$. In addition, approximately $40 \%$ clinical cases develop rapidly and suffer from recurrence and/or metastasis with fewer available treatments [4-7]. Therefore, it is greatly needed to find novel molecular targets and subsequently provide effective therapeutic strategies for the management of osteosarcoma.

Protein kinase B (PKB/AKT), a conserved serine/threonine protein kinase, is a nodal signaling effector for cell survival and proliferation and serves as an important therapeutic target for osteosarcoma $[8,9]$. Emerging studies have validated that AKT activation is sufficient to promote the growth, metastasis, and tumor-mediated bone destruction of osteosarcoma [10-12]. In addition, Shen et al. revealed that AKT activation by long noncoding RNA lncARSR also conferred chemoresistance to adriamycin and facilitated osteosarcoma progression [13]. Conversely, AKT inhibition significantly suppressed osteosarcoma cell proliferation and the malignant phenotype $[14,15]$. It is well-accepted that AKT is primarily dephosphorylated and inactivated by phosphatase and tensin homolog deleted on chromosome ten (PTEN), a major tumor suppressor gene in humans [16-18]. Moreover, inhibiting PTEN activity was capable of promoting osteosarcoma progression through activating AKT pathway [19]. Based on these findings, it is reasonable to target PTEN/ AKT pathway for the treatment of osteosarcoma.

MicroRNAs (miRNAs) are a class of short noncoding RNAs that post-transcriptionally regulate gene expression 
through binding to the $3^{\prime}$ - untranslated region (UTR) of target mRNAs [20-22]. Numerous miRNAs have been reported to be implicated in the progression of osteosarcoma and accurately predict the long-term outcome of osteosarcoma patients. Zhu et al. found that miR-23b-3p was significantly upregulated in osteosarcoma cells and that knockdown of miR-23b-3p shifted glycolysis to oxidative phosphorylation of osteosarcoma cells, thereby inhibiting osteosarcoma cell proliferation [23]. Results from Liu et al. indicated that miR210-5p was upregulated in clinical osteosarcoma specimens and cell lines and that miR-210-5p overexpression facilitated epithelial-mesenchymal transition and oncogenic autophagy, causing osteosarcoma overgrowth and pulmonary metastasis [24]. miR-181a-5p plays critical roles in regulating cell survival and proliferation, and aberrant miR-181a-5p expression has been linked with the progression of multiple cancers [25-27]. In addition, Xia et al. determined that miR-181a-5p expression in peripheral blood and cartilage tissues was decreased in osteoarthritis patients and that the inhibition of miR-181a-5p significantly increased tumor necrosis factor $\alpha$ and aggravated osteoarthritis [28]. In the present study, we aim to investigate the function and potential molecular basis of miR-181a-5p in osteosarcoma.

\section{Materials and Methods}

2.1. Cell Cultures and Treatments. The human osteosarcoma cells SaOS2, MG63, HOS, 143B and U2OS, and a human osteoblast cell hFOB1.19 were purchased from Type Culture Collection of the Chinese Academy of Sciences (Shanghai, China) and cultured in DMEM medium containing $10 \%$ fetal bovine serum (FBS) and $1 \%$ penicillin/streptomycin at $37^{\circ} \mathrm{C}$ in a $5 \%$ $\mathrm{CO}_{2}$ incubator. Cells were incubated with the mimic, inhibitor of miR-181a-5p, or their negative control (NC) at a concentration of $100 \mathrm{nmol} / \mathrm{L}$ for $24 \mathrm{~h}$ using Lipofectamine 6000 reagent (Beyotime; Shanghai, China), and then cultured in fresh medium supplemented with $10 \%$ FBS for additional $96 \mathrm{~h}$ [29]. miR-181a-5p mimic (miR10000256-1-5), inhibitor (miR20000256-1-5), and the NC were all purchased from Guangzhou RiboBio Co., Ltd. (Guangzhou, China). To clarify the role of PTEN, cells were infected with adenovirus carrying the full-length PTEN (Ad-PTEN) or green fluorescent protein (Ad-GFP) at a multiplicity of infection of 30 for $4 \mathrm{~h}$ and maintained in fresh medium for additional $24 \mathrm{~h}$ before miR-181a$5 \mathrm{p}$ mimic treatment. The Ad-PTEN and Ad-GFP were all generated by Hanbio Biotechnology Co., Ltd. (Shanghai, China). To inhibit AKT, cells were treated with AKT kinase inhibitor $(1 \mu \mathrm{mol} / \mathrm{L})$ for $24 \mathrm{~h}$ before miR-181a-5p mimic treatment [30]. To investigate whether miR-181a-5p mimic treatment would further inhibit growth of osteosarcoma cells in the presence of chemotherapeutic reagents, cells were pretreated with $0.4 \mu \mathrm{mol} / \mathrm{L}$ adriamycin (ADR; Sigma-Aldrich, St. Louis, MO, USA) and $1 \mu \mathrm{mol} / \mathrm{L}$ cisplatin (DDP, Sigma-Aldrich) for $24 \mathrm{~h}$ before miR-181a-5p mimic treatment according to previous studies $[31,32]$.

2.2. Cell Viability and Colony Formation Assay. Cell viability was determined using cell count kit-8 (CCK-8) kit (Beyotime, Shanghai, China) [33-36]. Briefly, cells were incubated in serum-free DMEM medium containing $10 \mu \mathrm{L}$ CCK-8 solution at $37^{\circ} \mathrm{C}$ for $2 \mathrm{~h}$ in the dark, and then the optical density (OD) value was detected at $450 \mathrm{~nm}$ using a microplate reader (Synergy HT, BioTek). To detect the colony formation, cells were treated and then cultured for addition 2 weeks with the medium replaced every 3 days. Next, cells were fixed with $4 \%$ paraformaldehyde at room temperature for $20 \mathrm{~min}$ and stained with $0.2 \%$ crystal violet (Wuhan Servicebio Technology Co., Ltd., Wuhan, China) at room temperature for $30 \mathrm{~min}$, followed by the rinse under tap water for additional $20 \mathrm{~min}$. Finally, colonies with the diameter $>0.05 \mathrm{~mm}$ were counted in a blinded manner [37-39].

2.3. Wound Healing and Transwell Experiments. Wound healing experiment was performed to evaluate the migrative capacity of osteosarcoma cells in vitro according to previous studies [40, 41]. In brief, cells were seeded to 6-well plates and allowed to grow to $\sim 90 \%$ confluence overnight. Then, a $200 \mu \mathrm{L}$ yellow pipette tip was used to scratch a gap on the cellular monolayers, and cells were then washed with phosphate buffer (PBS) to remove the suspended cells, followed by the incubation in serum-free DMEM medium for additional $24 \mathrm{~h}$. Images were captured using an optical microscope and the gap sized was recorded. To determine the invasive capacity of osteosarcoma cells, transwell experiment was performed as previously described [42-44]. Briefly, cells at a density of $2 \times 10^{5}$ per well were seeded onto the upper chambers that were precoated with polymerized matrigel, and the lower chambers were filled with $800 \mu \mathrm{L}$ DMEM medium containing $20 \%$ FBS as a chemotactic factor. $48 \mathrm{~h}$ later, cells in the upper surfaces were removed and cells in the bottom of the filters were stained with $0.2 \%$ crystal violet at room temperature for $30 \mathrm{~min}$. The invasive cells were then counted in a blinded manner.

2.4. Cell Cycle Analysis. Cell cycle distribution was analyzed by a flow cytometry using propidium iodide (PI) staining method $[45,46]$. Briefly, cells were stained with $0.5 \mathrm{~mL}$ PI/ RNase Staining Buffer (BD Bioscience, Franklin, NJ, USA) at $37^{\circ} \mathrm{C}$ for $15 \mathrm{~min}$ after being fixed in $70 \%$ ethanol at $-20^{\circ} \mathrm{C}$ overnight, and then were subjected to flow cytometry analysis to detect the fractions of the cells in G0/G1, S, and G2/M phase using FACSCalibur flow cytometer (BD Bioscience).

2.5. Western Blot. Cells were washed by PBS for 3 times and then lysed in RIPA lysis buffer (Wuhan Servicebio Technology Co., Ltd.) with the protein concentration measured by a Pierce $^{\mathrm{TM}}$ Microplate BCA Protein Assay Kit (Invitrogen, Carlsbad, CA, USA) [47-49]. Next, equal amounts of total proteins were separated by sodium dodecyl sulfate-polyacrylamide gel electrophoresis and then transferred onto polyvinylidene fluoride membrane and followed by the incubation in $5 \%$ skimmed milk at room temperature for additional $1 \mathrm{~h}$ to block the nonspecific binding of primary antibodies. After that, the membranes were incubated with indicated primary antibodies at $4^{\circ} \mathrm{C}$ overnight and the secondary antibody at room temperature for additional $1 \mathrm{~h}$ on the second day. Finally, the bands were visualized using electrochemiluminescence reagent and 


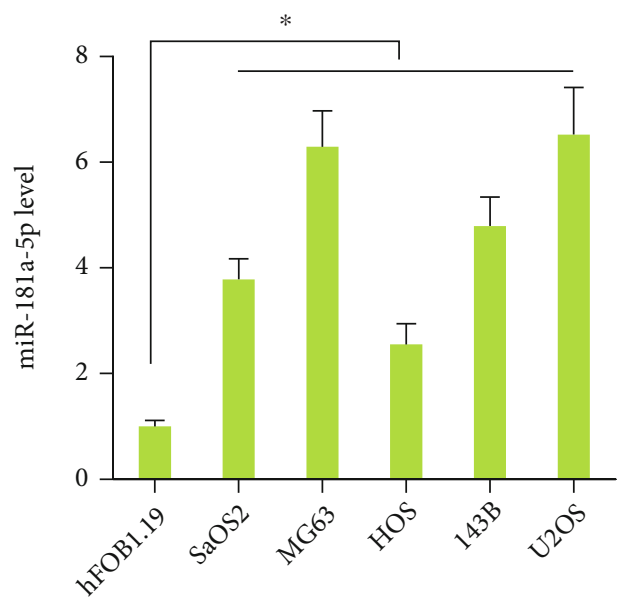

(a)

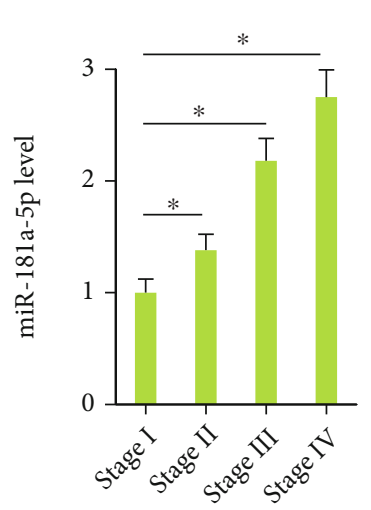

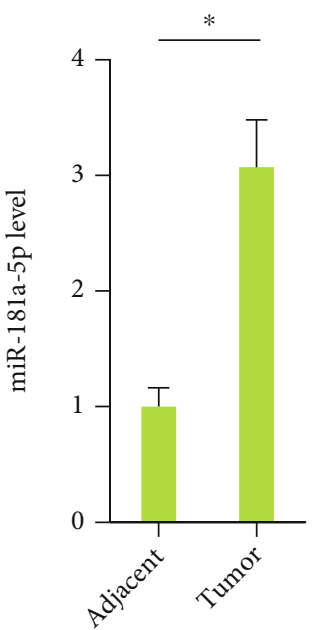

(b)

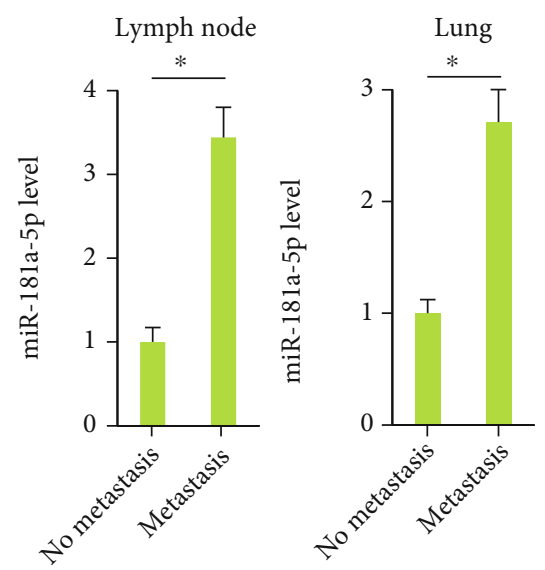

(c)

FIGURE 1: miR-181a-5p level is elevated in human osteosarcoma cells and tissues. (a) Relative miR-181a-5p level in different osteosarcoma cells and healthy human osteoblast cell $(n=6)$. (b) Relative miR-181a-5p level in human osteosarcoma and adjacent normal samples $(n=9$ 6). (c) Relative miR-181a-5p level in human osteosarcoma samples was analyzed according to the tumor stage and metastasis status $(n=12)$. All data are presented as the mean $\pm \mathrm{SD},{ }^{*} P<0.05$ versus the matched group.

analyzed by Image Lab software (Version 6.0, Bio-Rad). The primary antibodies against phospho-KT (p-AKT), total-AKT (t-AKT), PTEN, and glyceraldehyde-3-phosphate dehydrogenase (GAPDH) were all purchased from Cell Signaling Technology (Danvers, MA, USA).

2.6. Reverse Transcription-Quantitative Polymerase Chain Reaction (RT-qPCR). The total RNA was extracted from cultured cells with TRIzol reagent (Invitrogen) and reversely transcribed to cDNA using the TaqMan ${ }^{\circledR}$ microRNA Reverse Transcription Kit (Applied Biosystems, Foster City, CA, USA) according to the manufacturer's instructions [50-52]. Then, the relative levels of miR-181a-5p were determined with the TaqMan microRNA Assays Kit (Applied Biosystems) and normalized to U6 using the $2^{-\Delta \Delta \mathrm{Ct}}$ method on a 2.1 Real-Time PCR System using Bio-Rad CFX Manager (Bio-Rad, USA). The sequences were provided as below: miR-181a-5p, forward, 5'-TAGAGCTAGCGAATTCTTT GTTGGAAGAATCATGCTTCT-3', reverse, 5' -TTGCGG
CCGCGGATCCCATTGTTCAGTGAGCTTGTCCAC-3' ; U6, forward, 5'-GCTTCGGCAGCACATATACTAAAAT-3', reverse, $5^{\prime}$-CGCTTCACGAATTTGCGTGTCAT-3'.

2.7. Luciferase Reporter Assay. The direct binding of miR$181 \mathrm{a}-5 \mathrm{p}$ to the $3^{\prime}$-UTR of PTEN was validated by the luciferase reporter assay as described previously [53, 54]. In brief, the wild-type (WT) PTEN 3' -UTR with position 2310-2316 or truncated (TNC) PTEN 3'-UTR without position 23102316 was cloned into the sites $3^{\prime}$ of the firefly luciferase gene (luc2) of pmirGLO luciferase reporter plasmids (Promega Corporation, Madison, WI, USA), which were then cotransfected with miR-181a-5p mimic or mimic-NC to the HEK293T cells using Lipofectamine 6000 reagent (Beyotime). $48 \mathrm{~h}$ later, the fluorescent changes were measured using the dual luciferase reporter assay system with the firefly luciferase activity normalized to the corresponding Renilla luciferase activity. 


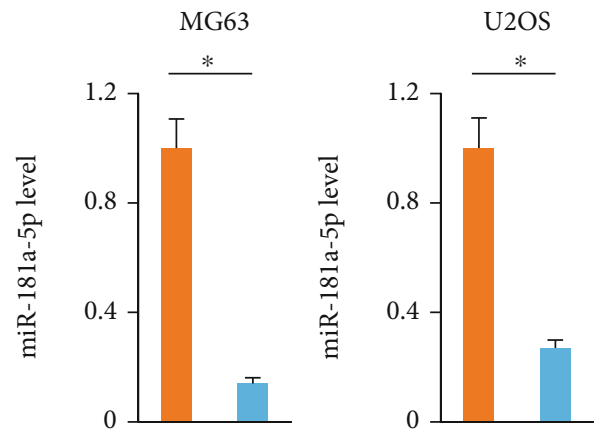

(a)
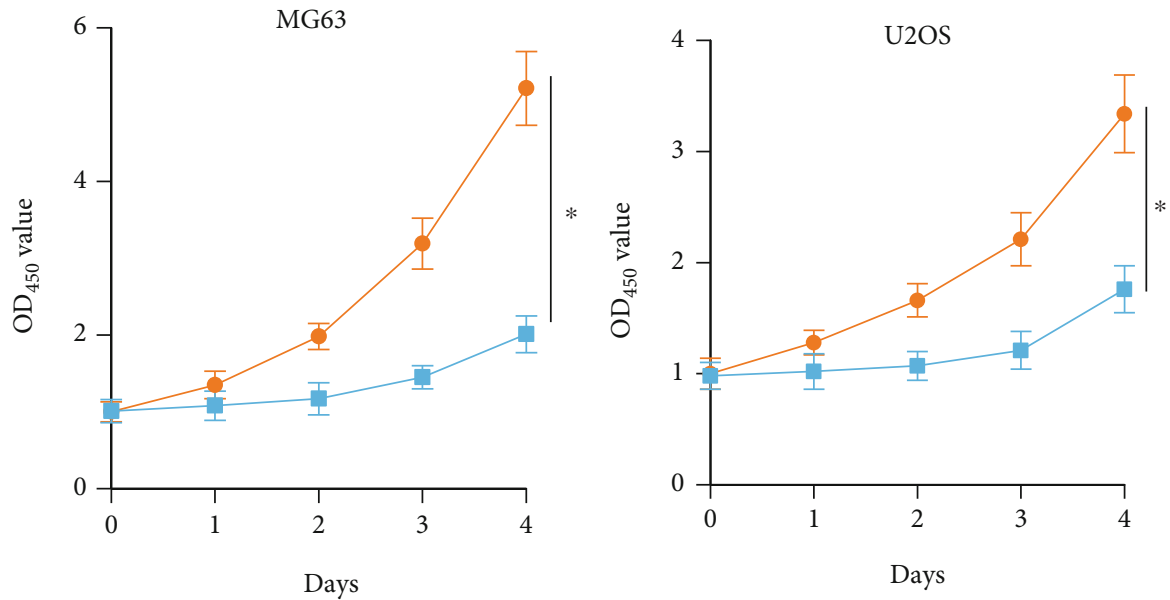

- - Inhibitor-NC

- Inhibitor

(b)

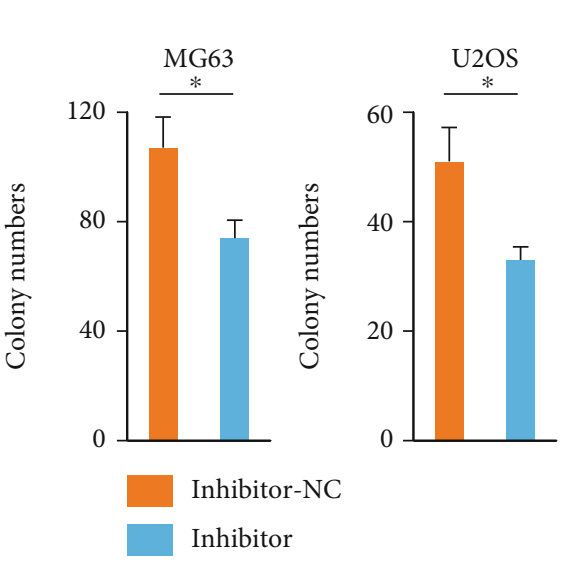

(c)
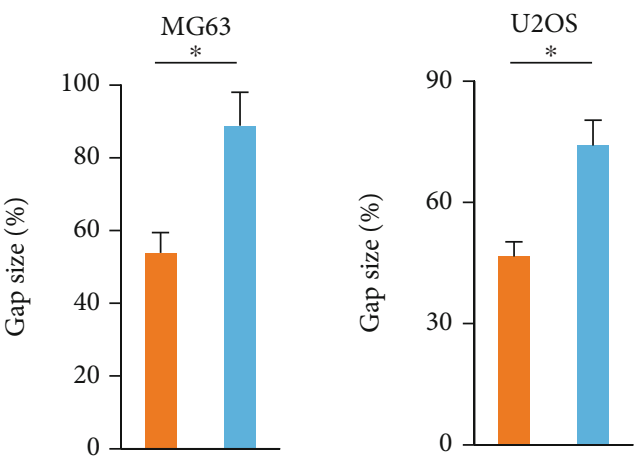

FIgUre 2: Continued.

(d) 


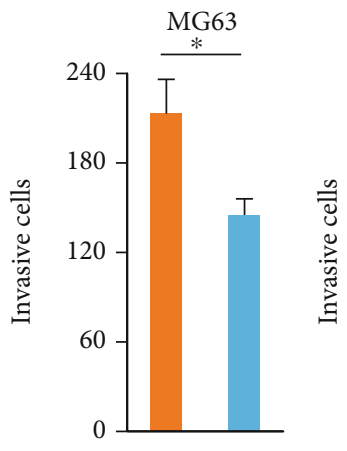

(e)
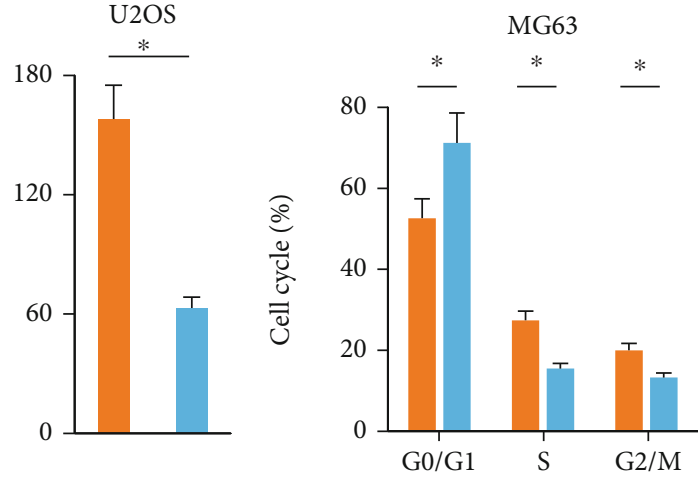

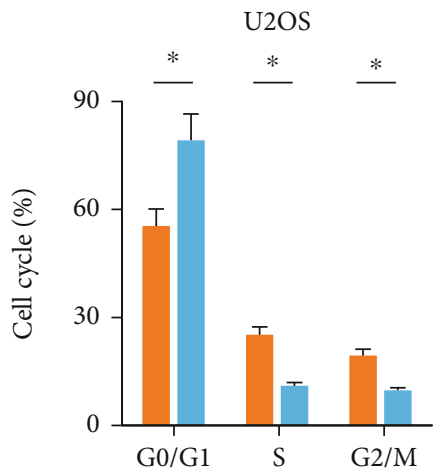

(f)

FIGURE 2: miR-181a-5p inhibitor restrains osteosarcoma progression in vitro. (a) Relative miR-181a-5p level in osteosarcoma cells treated with or without miR-181a-5p inhibitor $(n=6)$. (b) Cell viability of osteosarcoma cells determined by CCK-8 assay $(n=6)$. (c) Colony numbers in osteosarcoma cells with or without miR-181a-5p inhibitor treatment $(n=6)$. (d) Gap size in the wound healing experiments $(n=5)$. (e) Invasive cells in transwell experiments with or without miR-181a-5p inhibitor treatment $(n=6)$. (f) Cell cycle distribution in different phases $(n=5)$. All data are presented as the mean $\pm \mathrm{SD},{ }^{*} P<0.05$ versus the matched group.

2.8. Human Samples. Human osteosarcoma and adjacent normal tissues were obtained from 96 osteosarcoma patients who did not undergo radiotherapy or chemotherapy before surgery, and their clinicopathological characteristics were provided in supplementary Table. All tissues were preserved in liquid nitrogen immediately after resection and stored at $-80^{\circ} \mathrm{C}$ before further detection. Written informed consent was obtained from the patients or their legal guardians in accordance with the guidelines of in the Declaration of Helsinki. All experimental procedures were approved by the institutional review board and the ethics committee of our hospitals (Approval No. syrmyy2019-174).

2.9. Statistical Analysis. The SPSS software (Version 22.0) was used for statistical analysis with the $P<0.05$ considered statistically significant. All data were presented as the mean $\pm S D$, and unpaired Student's $t$-test test and one-way ANOVA followed by Tukey's post hoc test were used for comparisons between 2 groups and among 3 or more groups.

\section{Results}

3.1. miR-181a-5p Level is Elevated in Human Osteosarcoma Cells and Tissues. First, we detected the level of miR-181a$5 \mathrm{p}$ in six human osteosarcoma cell lines and a human osteoblast cell hFOB1.19. As shown in Figure 1(a), miR181a-5p level was significantly elevated in human osteosarcoma cells, especially the MG63 and U2OS cells. We then examined the expression of miR-181a-5p in 24 paired osteosarcoma tissues and adjacent normal tissues and detected a remarkable elevation of miR-181a-5p in human osteosarcoma tissues compared with healthy tissues (Figure 1(b)). In addition, increased miR-181a-5p level positively correlated with the advanced TNM stage, lymph node, and lung metastasis in osteosarcoma patients (Figure 1(c)). Together, these data indicate that miR-181a-5p may be implicated in the progression of human osteosarcoma.

\subsection{Mir-181a-5p Inhibitor Restrains Osteosarcoma} Progression In Vitro. In the above study, MG63 and U2OS cells were found to have higher miR-181a-5p expression than other osteosarcoma cells; therefore, we used these two cells in our further study. To clarify the role of miR-181a$5 p$ in osteosarcoma cells, MG63 and U2OS cells were transfected with miR-181a-5p inhibitor to reduce the expression of endogenous miR-181a-5p (Figure 2(a)). As determined by the CCK- 8 assay, we found that miR-181a-5p inhibitor significantly reduced human osteosarcoma cell viability in vitro (Figure 2(b)). As shown in Figure 2(c), the cells treated with miR-181a-5p inhibitor also had decreased colony formative capacity. The above-mentioned study (Figure 1(c)) showed that miR-181a-5p level positively correlated with the advanced TNM stage, lymph node, and lung metastasis in osteosarcoma patients. Migration and invasion are two determinants of the malignant osteosarcoma phenotype and are associated with the local bone destruction and distant metastasis $[55,56]$. Wound-healing and transwell experiments were performed to evaluate the migrative and invasive capacities of osteosarcoma cells. As expected, miR181a-5p inhibitor significantly suppressed MG63 and U2OS cells migration and invasion (Figures 2(d) and 2(e)). In addition, the percentage of G1/G0 phase cells was increased, while the percentage of $S$ and $\mathrm{G} 2 / \mathrm{M}$ phase cells was decreased in miR-181a-5p inhibitor-treated osteosarcoma cells (Figure 2(f)). Collectively, these findings suggest that miR-181a-5p inhibitor suppresses the growth, colony formation, migration and invasion of osteosarcoma cells in vitro, and induces G1/G0 cell cycle arrest.

\subsection{Mir-181a-5p Mimic Promotes Osteosarcoma Progression In} Vitro. Then, MG63 and U2OS cells were treated with miR181a-5p mimic to investigate whether overexpression could promote osteosarcoma progression in vitro (Figure 3(a)). As shown in Figures 3(b) and 3(c), miR-181a-5p mimic significantly increased MG63 and U2OS cells viability and colony formation. And their migrative and invasive capacities were 

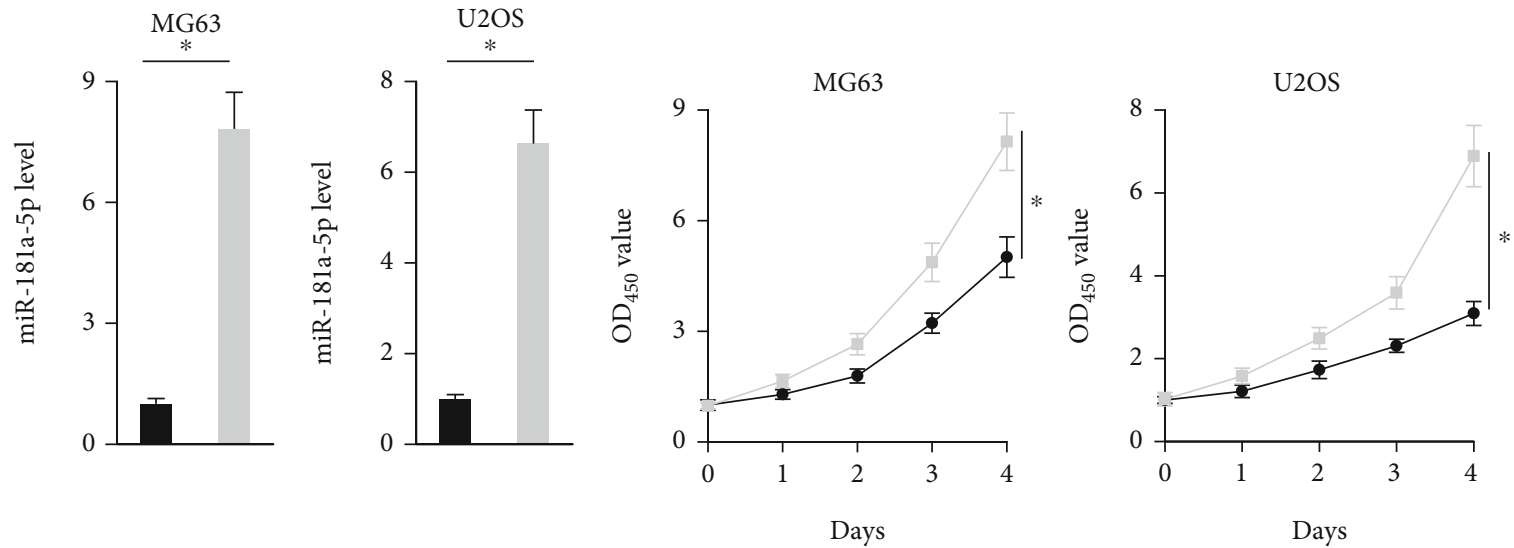

- Mimic-NC
- Mimic

(a)

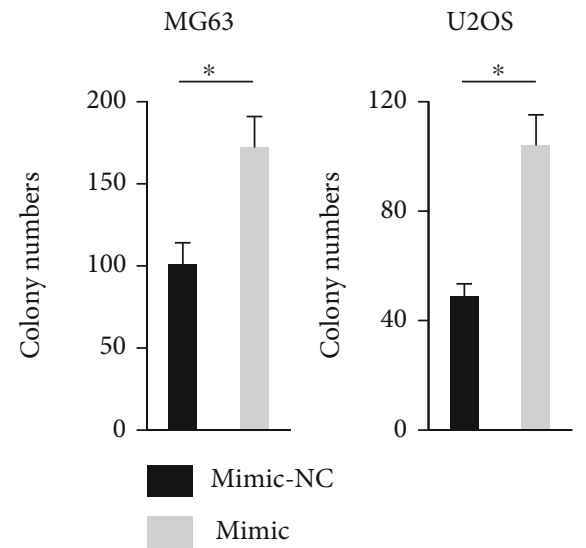

(c)

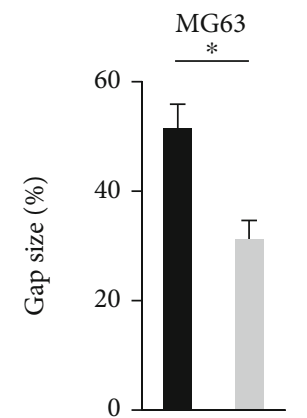

(b)

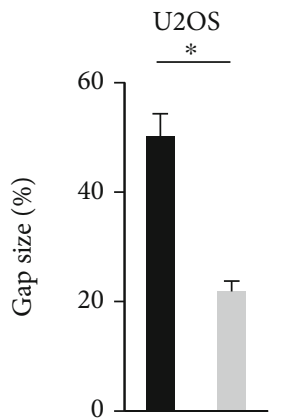

(d)
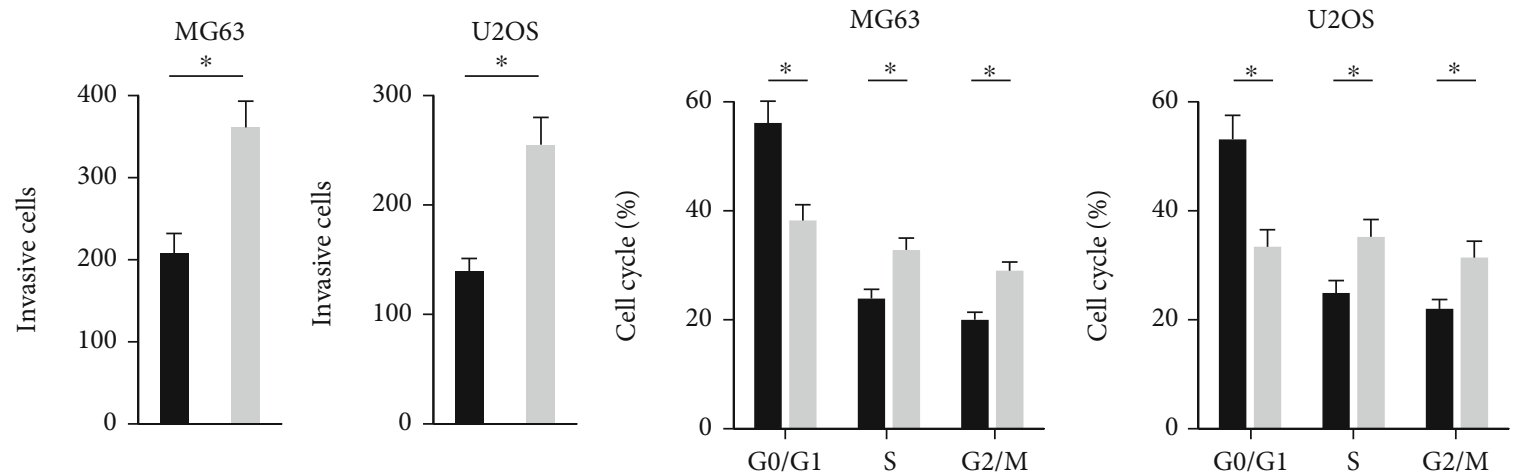

(e)

(f)

FIGURE 3: miR-181a-5p mimic promotes osteosarcoma progression in vitro. (a) Relative miR-181a-5p level in osteosarcoma cells treated with or without miR-181a-5p mimic $(n=6)$. (b) Cell viability of osteosarcoma cells determined by CCK-8 assay ( $n=6)$. (c) Colony numbers in osteosarcoma cells with or without miR-181a-5p mimic treatment $(n=6)$. (d) Gap size in the wound healing experiments $(n=5)$. (e) Invasive cells in transwell experiments with or without miR-181a-5p mimic treatment $(n=6)$. (f) Cell cycle distribution in different phases $(n=5)$. All data are presented as the mean $\pm \mathrm{SD},{ }^{*} P<0.05$ versus the matched group.

also enhanced by miR-181a-5p mimic treatment, indicating an increased malignance of the MG63 and U2OS cells (Figures 3(d) and 3(e)). In addition, miR-181a-5p mimic also significantly decreased the cell numbers in G0/G1 phase yet increased the cells in $\mathrm{S}$ and G2/M phase (Figure 3(f)). In addi- tion, we also examined the role of miR-181a-5p mimic in HOS cell with low expression of miR-181a-5p. As shown in Supplementary Figure $1 \mathrm{~A}-1 \mathrm{E}$, miR-181a-5p mimic significantly promoted the proliferation, colony formation, migration ,and invasion of HOS cell in vitro. These data reveal that 


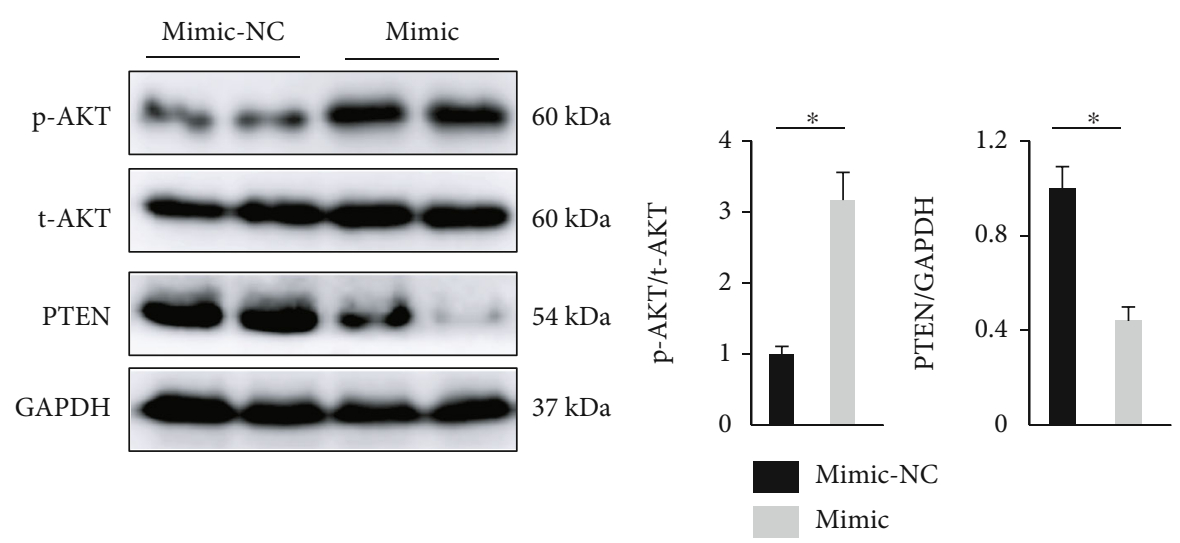

(a)

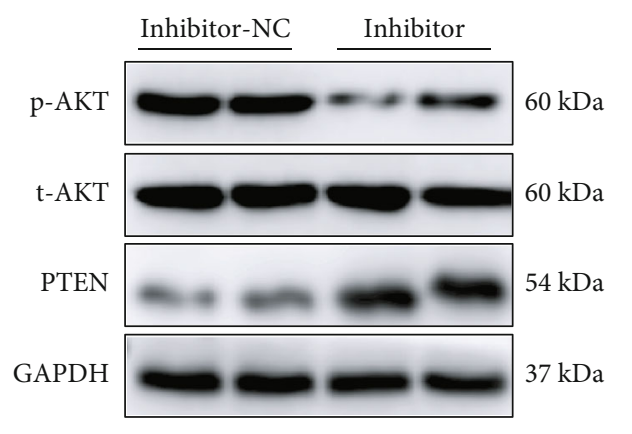

(b)

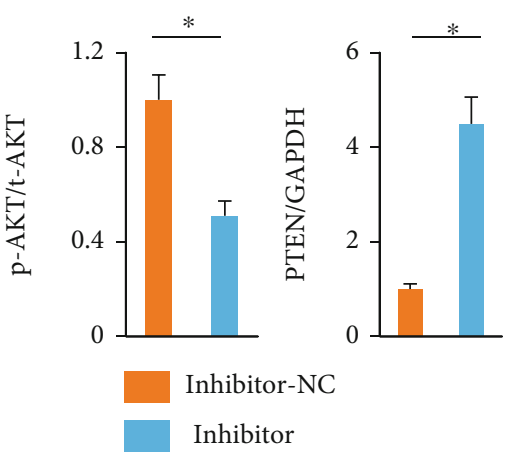

Inhibitor

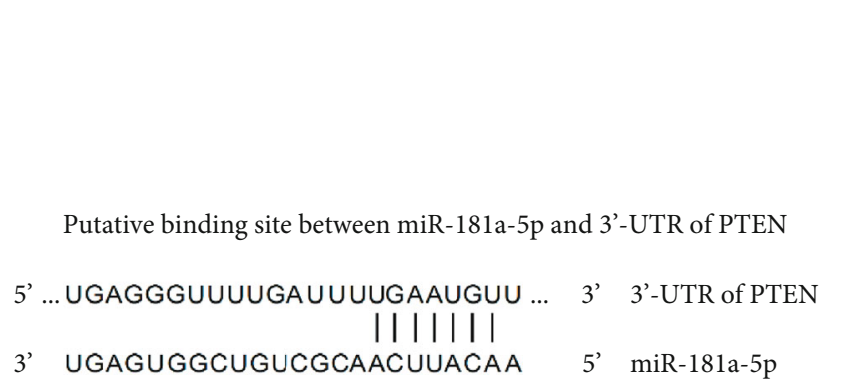

(d)

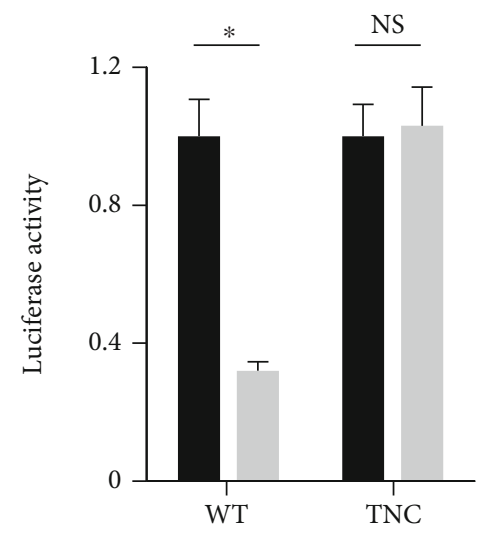

(e)

FIgUre 4: Continued. 


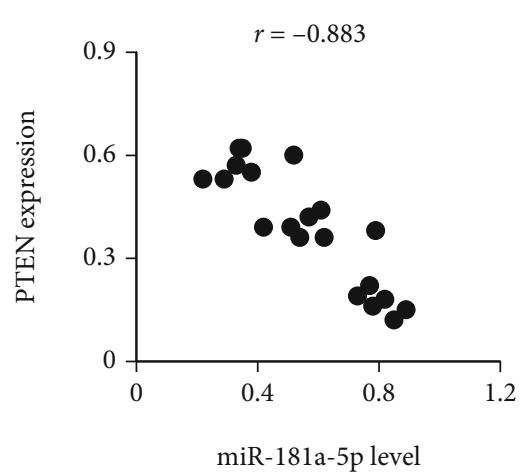

(f)

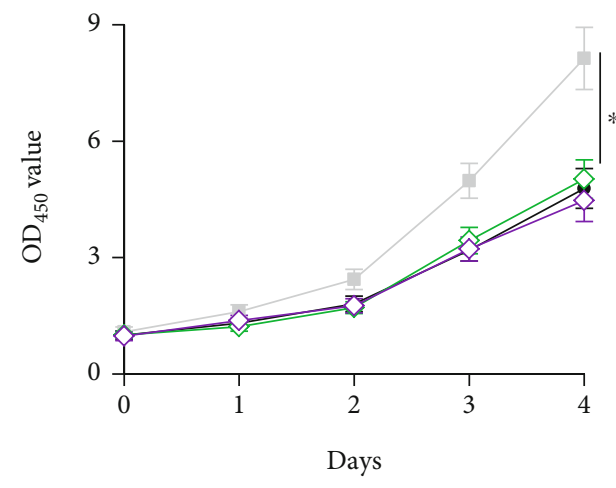

$\multimap$ Mimic-NC $\checkmark$ Mimic + AKTi

Mimic $\prec$ Mimic+Ad-PTEN

(h)

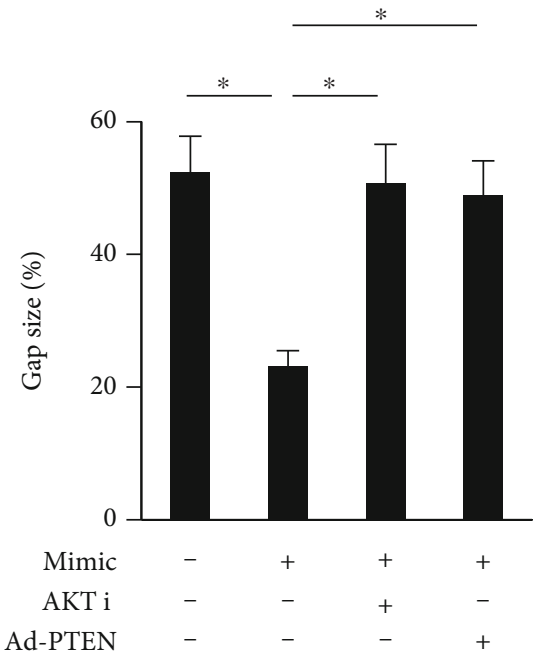

(j)

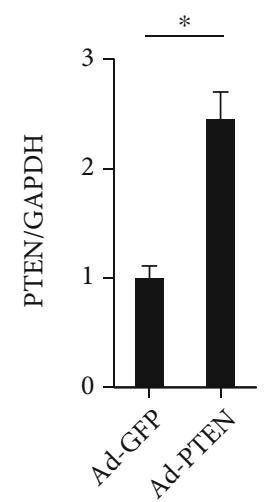

(g)

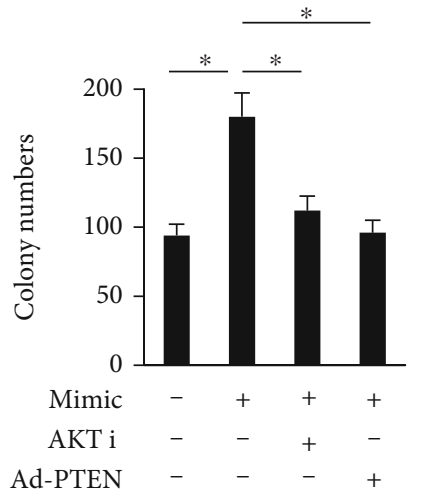

(i)

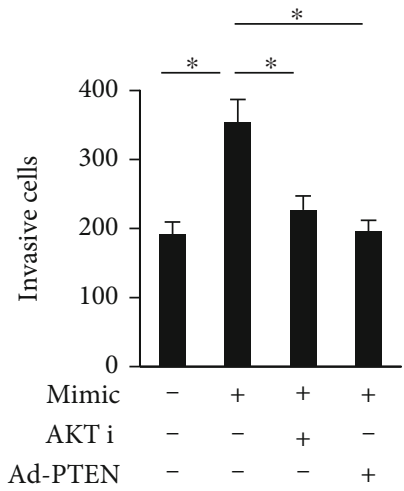

(k)

Figure 4: Continued. 


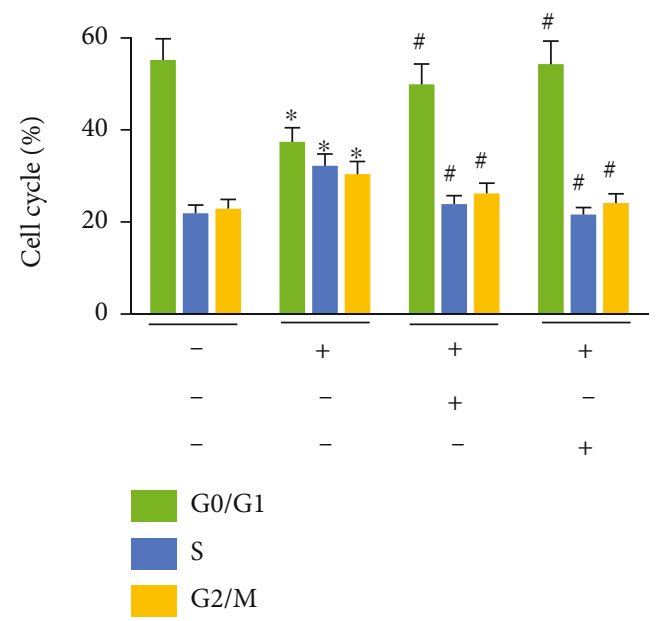

(1)

FIGURE 4: miR-181a-5p modulates osteosarcoma progression via PTEN-AKT pathway in vitro. (a and b) Representative western blot images and the statistical analysis of AKT and PTEN $(n=6)$. (c) Relative PTEN mRNA levels in osteosarcoma cells treated with miR-181a-5p mimic or inhibitor $(n=6)$. (d) Putative binding site of between miR-181a-5p and $3^{\prime}$-UTR of PTEN. (e) Relative luciferase activity in HEK293T cells $(n=6)$. (f) Correlation between miR-181a-5p level and PTEN expression in human osteosarcoma tissues $(n=20)$. (g) Representative western blot images and the statistical analysis of PTEN $(n=6)$. (h and i) Cell viability and colony numbers in osteosarcoma cells $(n=6)$. ( $\mathrm{j}$ and $\mathrm{k}$ ) Gap size and invasive cells in osteosarcoma cells $(n=6)$. (l) Cell cycle distribution in different phases $(n=5)$. All data are presented as the mean $\pm \mathrm{SD},{ }^{*} P<0.05$ versus the matched group. $(1){ }^{*} P<0.05$ versus control group, ${ }^{\#} P<0.05$ versus miR-181a-5p mimic-treated osteosarcoma cells.

miR-181a-5p mimic contributes to the proliferation, colony formation, migration, invasion, and cell cycle progression of osteosarcoma cells in vitro.

3.4. Mir-181a-5p Modulates Osteosarcoma Progression via PTEN-AKT Pathway In Vitro. AKT is a nodal signaling effector for cell survival and proliferation and serves as an important therapeutic target for osteosarcoma $[14,15]$. We then investigated whether miR-181a-5p modulates osteosarcoma progression through AKT. As shown in Figures 4(a) and 4(b), AKT in MG63 cells was activated by miR-181a$5 \mathrm{p}$ mimic yet inhibited by miR-181a-5p inhibitor. PTEN is a major tumor suppressor gene in humans and functions as a classic upstream phosphatase of AKT pathway. Intriguingly, we found that PTEN mRNA and protein levels were decreased in miR-181a-5p mimic-treated MG63 cells, but increased in miR-181a-5p inhibitor-treated MG63 cells (Figures $4(\mathrm{a})-4(\mathrm{c}))$. In addition, a putative binding site of miR-181a-5p was observed in the $3^{\prime}$-UTR of PTEN using the TargetScan online software (Figure 4(d)). To validate the prediction, luciferase reporter assay was performed. The data suggested that miR-181a-5p mimic could directly inhibit the luciferase activity in HEK293T cells transfected with WT 3 '-UTR of PTEN but not the TNC form (Figure 4(e)). Meanwhile, we observed a negative correlation between miR-181a-5p level and PTEN expression in human osteosarcoma tissues (Figure 4(f)). Next, we overexpressed PTEN in MG63 cells with the adenovirus and the efficiency was validated by the western blot (Figure $4(\mathrm{~g})$ ). As shown in Figures 4(h) and 4(i), miR-181a-5p mimic significantly increased the viability and colony formation in MG63 cells, which were almost completely abolished by either PTEN overexpression or AKT inhibition. And the increased migra- tive and invasive capacities seen in miR-181a-5p mimictreated MG63 cells were also decreased by either PTEN overexpression or AKT inhibition (Figures 4(j) and 4(k)). In addition, both PTEN overexpression and AKT inhibition could significantly blocked miR-181a-5p mimic-mediated cell cycle progression in MG63 cells (Figure 4(1)). Taken together, our findings identify that miR-181a-5p modulates osteosarcoma progression via PTEN-AKT pathway in vitro.

3.5. Mir-181a-5p Inhibitor Further Inhibits Growth of Osteosarcoma In Vitro in the Presence of Classic Chemotherapeutic Agents. Since miR-181a-5p inhibitor significantly restrained osteosarcoma progression in vitro, we then determined whether miR-181a-5p inhibitor could further inhibit growth of human osteosarcoma cells in the presence of ADR or DDP, two common chemotherapeutic agents for the treatment of osteosarcoma. As shown in Figures 5(a) and 5(b), ADR incubation significantly inhibited the viability and colony formation in human osteosarcoma cells, which were further enhanced in the presence of miR-181a-5p inhibitor. As expected, miR-181a-5p inhibitor also further inhibited growth of DDP-treated MG63 and U2OS cells, as determined by the decreased cell viability and colony formation (Figures 5(c) and 5(d)). Collectively, the results approve that miR-181a-5p inhibitor can further inhibit growth of osteosarcoma in vitro in the presence of classic chemotherapeutic agents, at least $\mathrm{ADR}$ and DDP.

\section{Discussion}

Osteosarcoma is identified as a malignant bone tumor in adolescents with a poor prognosis due to pulmonary metastasis. Given the deeper understanding of the pathophysiological 


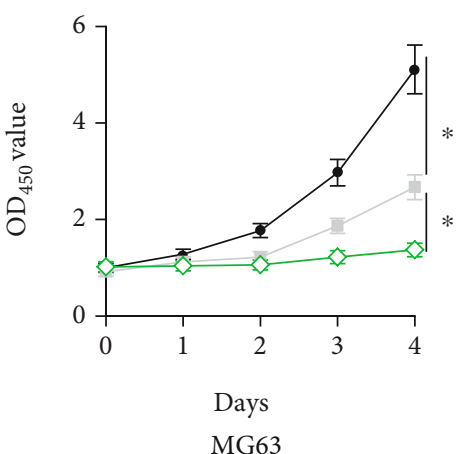

MG63

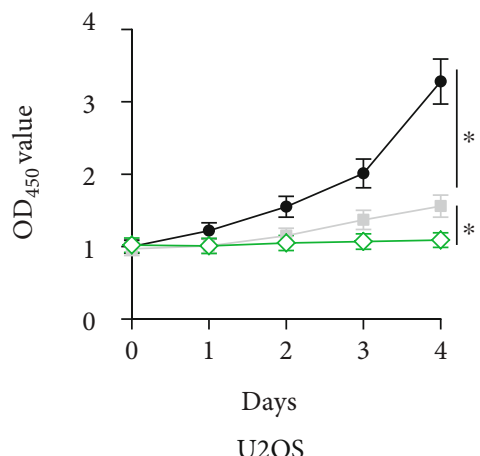

$\mathrm{U} 2 \mathrm{OS}$

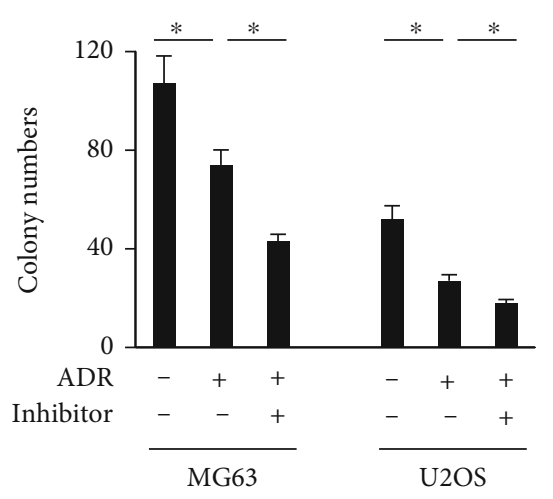

(a)
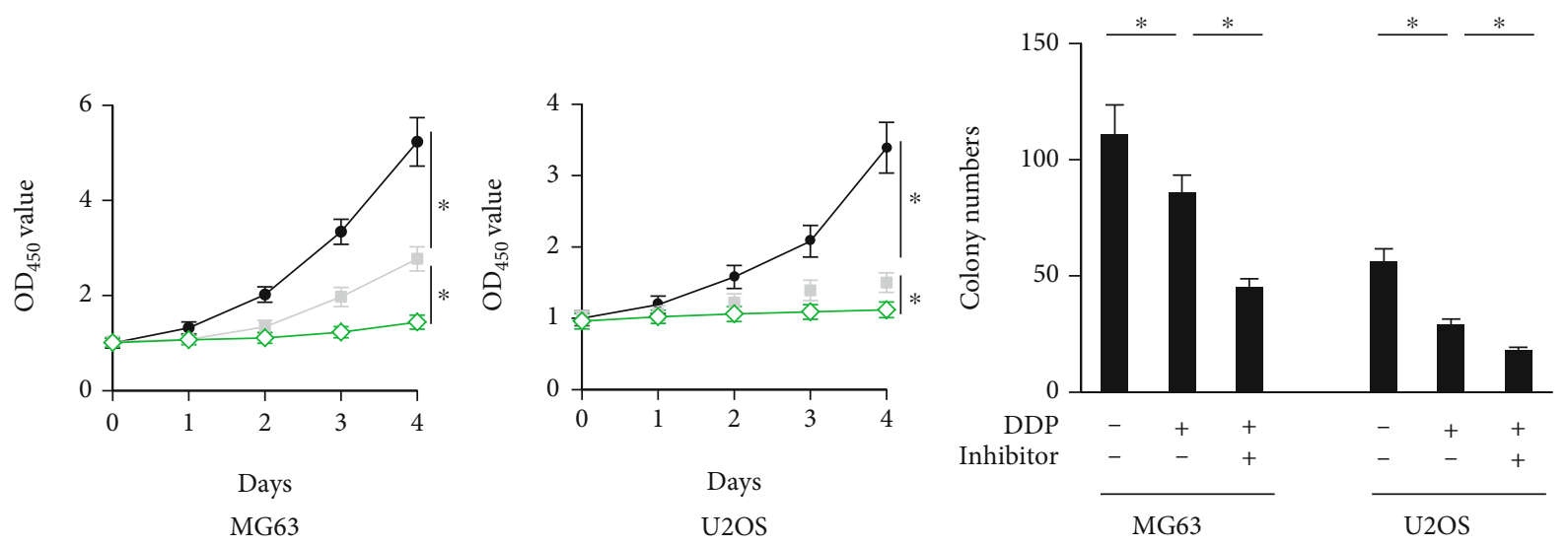

(c)

FIGURE 5: miR-181a-5p inhibitor further inhibits growth of osteosarcoma in vitro in the presence of classic chemotherapeutic agents. (a) Cell viability of osteosarcoma cells determined by CCK-8 assay $(n=6)$. (b) Colony numbers in ADR-treated osteosarcoma cells with or without miR-181a-5p inhibitor incubation $(n=6)$. (c) Cell viability of osteosarcoma cells determined by CCK- 8 assay $(n=6)$. (d) Colony numbers in DDP-treated osteosarcoma cells with or without miR-181a-5p inhibitor incubation $(n=6)$. All data are presented as the mean \pm SD, ${ }^{*} P<0.05$ versus the matched group.

mechanism and rapid progress of medical treatment for osteosarcoma, the 5-year survival rate in nonmetastatic patients has reached from $20 \%$ to $60 \%-70 \%$ in the past 30 years; however, approximately $40 \%$ clinical cases suffer from recurrence and/ or metastasis with very low survival rate $[2,3,7]$. In the present study, we reveal that miR-181a-5p level is elevated in human osteosarcoma cells and tissues, and positively correlates with the advanced TNM stage, lymph node, and lung metastasis in clinical cases. miR-181a-5p mimic contributes to, while miR181a-5p inhibitor suppresses the proliferation, colony formation, migration, invasion, and cell cycle progression of osteosarcoma cells in vitro through regulating PTEN-AKT pathway. More importantly, miR-181a-5p inhibitor can further inhibit growth of human osteosarcoma cells in the presence of chemotherapeutic agents (e.g. ADR and DDP). Collectively, our data determine that the increased miR-181a-5p level in osteosarcoma is essential for its development and the malignant poten- tial, and that inhibiting miR-181a-5p may provide novel therapeutic strategies to control osteosarcoma.

Emerging evidences have verified that the aberrant miRNAs are implicated in the progression of osteosarcoma via regulating multiple biological processes, including growth, migration, and invasion and cell cycle, miR-181a-5p has been linked with various human cancers, including breast cancer, prostate cancer, and melanoma and non-small-cell lung cancer. Benedetti et al. demonstrated that miR-181a$5 \mathrm{p}$ was downregulated in human breast cancer samples and could be used as a promising biomarker for prognosis of primary breast cancer [57]. Results from Wang et al. indicated that serum miR-181a-5p was significantly elevated in prostate cancer patients with bone metastasis than those in non-bone metastatic prostate cancer groups and could predict the risk of bone metastasis for prostate cancer patients [58]. In a previous study, the authors showed that miR- 
181a-5p directly targeted and inhibited BCL-2, thereby reducing melanoma stem cells apoptosis and promoting the tumorigenesis of melanoma in vivo [59]. In contrast, $\mathrm{Li}$ et al. demonstrated that miR-181a-5p suppression by a long noncoding RNA SNHG7 facilitated the proliferation, migration, and invasion of non-small-cell lung cancer [60]. Mutlu and colleagues detected a significant upregulation of miR181a-5p in human chondrosarcoma and furthermore, Xiong et al. identified a dysregulated miR-181a-5p expression between metastatic and non-metastatic osteosarcoma [61, 62]. However, relative little is known about the role of miR-181a-5p in osteosarcoma progression. Herein, we identified an oncologic role of miR-181a-5p in human osteosarcoma, and miR-181a-5p inhibitor significantly suppressed the proliferation, colony formation, migration, invasion and cell cycle progression, and enhanced the chemosensitivity of osteosarcoma cells in vitro.

$\mathrm{AKT}$, a multifunctional kinase, plays critical roles in regulating cell growth and is constitutively activated in various human cancers, including osteosarcoma [14, 63]. AKT directly phosphorylates and activates mammalian target of rapamycin (mTOR), a crucial signaling nexus to orchestrate protein synthesis and cell growth [64]. Wang et al. demonstrated that AKT-mediated activation of mTOR in osteosarcoma cells significantly upregulated glycolysis-related proteins and migration-related proteins, thereby facilitating osteosarcoma progression [65]. Xiao et al. showed that the activation of AKT-mTOR axis promoted autophagy and led to osteosarcoma chemoresistance [66]. Zhang and colleagues previously validated that AKT activation resulted in increased phosphorylation and nuclear translocation of nuclear factor kappa-B, eventually enhancing epithelialmesenchymal transition and migration of MG-63 osteosarcoma cells [67]. Under physiological conditions, AKT is dephosphorylated and inactivated by PTEN through the lipid phosphatase activity, a classical tumor suppressor gene in humans [14]. Herein, we disclosed that miR-181a-5p directly bound to the $3^{\prime}$-UTR of PTEN and reduced PTEN protein expression, thereby promoting AKT activation and osteosarcoma progression.

In summary, our data for the first time reveal that miR181a-5p promotes osteosarcoma progression via PTEN/ AKT pathway and that miR-181a-5p is a promising therapeutic target to treat osteosarcoma.

\section{Data Availability}

The data that support the findings of this study are available from the corresponding author upon reasonable request.

\section{Conflicts of Interest}

The authors declare that they have no competing interests.

\section{Authors' Contributions}

CS, FA, LX, and XG designed the experiments; CS, CC, and ZC carried out the experiments; CS, CC, JG, and ZHY analyzed the experimental results and interpreted the data; CS,
CC, FA, LX, and XG wrote and revised the manuscript. Chen Sun, Chi Chen, and Zhen Chen. These authors contributed equally to this work.

\section{Acknowledgments}

The present study was supported by the Health and Family Planning Commission of Wuhan Municipality (grant no. WX19Q15).

\section{Supplementary Materials}

Table S1: clinicopathological characteristics of 96 osteosarcoma patients; Figure S1: miR-181a-5p mimic promotes osteosarcoma progression in vitro. (Supplementary Materials)

\section{References}

[1] J. Li, X. Yan, J. Tang et al., "HDAC2-mediated upregulation of IL-6 triggers the migration of osteosarcoma cells," Cell Biology and Toxicology, vol. 35, no. 5, pp. 423-433, 2019.

[2] H. J. Siegel and J. G. Pressey, "Current concepts on the surgical and medical management of osteosarcoma," Expert Review of Anticancer Therapy, vol. 8, no. 8, pp. 1257-1269, 2008.

[3] M. Kansara, M. W. Teng, M. J. Smyth, and D. M. Thomas, "Translational biology of osteosarcoma," Nature Reviews. Cancer, vol. 14, no. 11, pp. 722-735, 2014.

[4] G. Ottaviani and N. Jaffe, "The epidemiology of osteosarcoma," Cancer Treatment and Research, vol. 152, pp. 3-13, 2009.

[5] S. Zheng, L. Wan, D. Ge et al., "LINC00266-1/miR-548c-3p/ SMAD2 feedback loop stimulates the development of osteosarcoma," Cell Death \& Disease, vol. 11, no. 7, p. 576, 2020.

[6] M. Gorska-Ponikowska, A. Ploska, D. Jacewicz et al., "Modification of DNA structure by reactive nitrogen species as a result of 2-methoxyestradiol-induced neuronal nitric oxide synthase uncoupling in metastatic osteosarcoma cells," Redox Biology, vol. 32, article 101522, 2020.

[7] B. Kempf-Bielack, S. S. Bielack, H. Jurgens et al., "Osteosarcoma relapse after combined modality therapy: an analysis of unselected patients in the cooperative osteosarcoma study group (COSS)," Journal of Clinical Oncology, vol. 23, no. 3, pp. 559-568, 2005.

[8] M. Li, C. Wu, J. S. Muhammad et al., "Melatonin sensitises shikonin-induced cancer cell death mediated by oxidative stress via inhibition of the SIRT3/SOD2-AKT pathway," Redox Biology, vol. 36, article 101632, 2020.

[9] V. S. Tomar, V. Patil, and K. Somasundaram, "Temozolomide induces activation of $\mathrm{Wnt} / \beta$-catenin signaling in glioma cells via PI3K/Akt pathway: implications in glioma therapy," Cell Biology and Toxicology, vol. 36, no. 3, pp. 273-278, 2020.

[10] Y. Zhang, H. Cheng, W. Li, H. Wu, and Y. Yang, "Highlyexpressed $\mathrm{P} 2 \mathrm{X} 7$ receptor promotes growth and metastasis of human HOS/MNNG osteosarcoma cellsviaPI3K/Akt/ GSK $3 \beta / \beta$-catenin and mTOR/HIF1 $\alpha /$ VEGF signaling," International Journal of Cancer, vol. 145, no. 4, pp. 1068-1082, 2019.

[11] Q. Zou, X. Xiao, Y. Liang et al., "miR-19a-mediated downregulation of RhoB inhibits the dephosphorylation of AKT1 and induces osteosarcoma cell metastasis," Cancer Letters, vol. 428, pp. 147-159, 2018. 
[12] G. Liu, X. Ouyang, Y. Sun et al., "The miR-92a-2-5p in exosomes from macrophages increases liver cancer cells invasion via altering the AR/PHLPP/p-AKT/ $\beta$-catenin signaling," Cell Death and Differentiation, vol. 27, no. 12, pp. 3258-3272, 2020.

[13] P. Shen and Y. Cheng, "Long noncoding RNA lncARSR confers resistance to Adriamycin and promotes osteosarcoma progression," Cell Death \& Disease, vol. 11, no. 5, p. 362, 2020.

[14] W. Han and J. Liu, "LncRNA-p21 inhibited the proliferation of osteosarcoma cells _via_the miR-130b/PTEN/AKT signaling pathway," Biomedicine \& Pharmacotherapy, vol. 97, pp. 911918, 2018.

[15] B. Li, J. Zhao, Q. Zhao et al., "MicroRNA-618 directly targets Metadherin mRNA to suppress the malignant phenotype of osteosarcoma cells by reducing PTEN-AKT pathway output," Oncotargets and Therapy, vol. 12, pp. 9795-9807, 2019.

[16] Q. Weng, M. Zhao, J. Zheng et al., "STAT3 dictates $\beta$-cell apoptosis by modulating PTEN in streptozocin-induced hyperglycemia," Cell Death and Differentiation, vol. 27, no. 1, pp. 130-145, 2020.

[17] X. Yun, Y. Fang, C. Lv et al., "Inhibition of the activation of $\gamma \delta$ T17 cells through PPAR $\gamma$ - PTEN/Akt/GSK3 $\beta /$ NFAT pathway contributes to the anti-colitis effect of madecassic acid," Cell Death \& Disease, vol. 11, no. 9, p. 752, 2020.

[18] L. Wang, X. Bo, X. Yi et al., "Exosome-transferred LINC01559 promotes the progression of gastric cancer via PI3K/AKT signaling pathway," Cell Death \& Disease, vol. 11, no. 9, p. 723, 2020.

[19] W. Wu, D. Jing, Z. Meng et al., "FGD1 promotes tumor progression and regulates tumor immune response in osteosarcoma via inhibiting PTEN activity," Theranostics, vol. 10, no. 6, pp. 2859-2871, 2020.

[20] Y. D. Jung, S. K. Park, D. Kang et al., "Epigenetic regulation of miR-29a/miR-30c/DNMT3A axis controls SOD2 and mitochondrial oxidative stress in human mesenchymal stem cells," Redox Biology, vol. 37, article 101716, 2020.

[21] Y. Jing, W. Liang, J. Liu et al., "Autophagy-mediating microRNAs in cancer chemoresistance," Cell Biology and Toxicology, vol. 36, no. 6, pp. 517-536, 2020.

[22] L. Chen, L. Chen, Z. Qin et al., "Upregulation of miR-489-3p and miR-630 inhibits oxaliplatin uptake in renal cell carcinoma by targeting OCT2," Acta Pharmaceutica Sinica B, vol. 9, no. 5, pp. 1008-1020, 2019.

[23] R. Zhu, X. Li, and Y. Ma, "miR-23b-3p suppressing PGC1alpha promotes proliferation through reprogramming metabolism in osteosarcoma," Cell Death \& Disease, vol. 10, no. 6, p. 381, 2019.

[24] W. Liu, D. Jiang, F. Gong et al., “miR-210-5p promotes epithelial-mesenchymal transition by inhibiting PIK3R5 thereby activating oncogenic autophagy in osteosarcoma cells," Cell Death \& Disease, vol. 11, no. 2, p. 93, 2020.

[25] X. Liang and W. Xu, "miR-181a-5p regulates the proliferation and apoptosis of glomerular mesangial cells by targeting KLF6," Experimental and Therapeutic Medicine, vol. 20, no. 2, pp. 1121-1128, 2020.

[26] Y. Zheng, B. Cai, X. Li, D. Li, and G. Yin, "MiR-125b-5p and miR-181b-5p inhibit keratinocyte proliferation in skin by targeting Akt3," European Journal of Pharmacology, vol. 862, article 172659, 2019.

[27] F. Tian, Y. Shen, Z. Chen, R. Li, J. Lu, and Q. Ge, “Aberrant miR-181b-5p and miR-486-5p expression in serum and tissue of non- small cell lung cancer," Gene, vol. 591, no. 2, pp. 338 343, 2016.

[28] S. Xia, H. Tian, L. Fan, and J. Zheng, "Peripheral blood miR181a-5p serves as a marker for screening patients with osteoarthritis by targeting TNFalpha," Clinical Laboratory, vol. 63, pp. 1819-1825, 2017.

[29] L. L. Lv, Y. Feng, M. Wu et al., "Exosomal miRNA-19b-3p of tubular epithelial cells promotes M1 macrophage activation in kidney injury," Cell Death and Differentiation, vol. 27, no. 1, pp. 210-226, 2020.

[30] Z. G. Ma, Y. P. Yuan, S. C. Xu et al., "CTRP3 attenuates cardiac dysfunction, inflammation, oxidative stress and cell death in diabetic cardiomyopathy in rats," Diabetologia, vol. 60, no. 6, pp. 1126-1137, 2017.

[31] M. Xian, H. Cao, J. Cao et al., "Bortezomib sensitizes human osteosarcoma cells to adriamycin-induced apoptosis through ROS-dependent activation of p-eIF2 $\alpha / \mathrm{ATF} 4 / \mathrm{CHOP}$ axis," International Journal of Cancer, vol. 141, no. 5, pp. 10291041, 2017.

[32] D. Yang, T. Xu, L. Fan, K. Liu, and G. Li, "microRNA-216b enhances cisplatin-induced apoptosis in osteosarcoma MG63 and SaOS-2 cells by binding to JMJD2C and regulating the HIF1 $\alpha /$ HES1 signaling axis," Journal of Experimental \& Clinical Cancer Research, vol. 39, no. 1, p. 201, 2020.

[33] C. Hu, X. Zhang, W. Wei et al., "Matrine attenuates oxidative stress and cardiomyocyte apoptosis in doxorubicin-induced cardiotoxicity via maintaining AMPK $\alpha$ /UCP2 pathway," Acta Pharmaceutica Sinica B, vol. 9, no. 4, pp. 690-701, 2019.

[34] Z. Guo, N. Tang, F. Y. Liu et al., “TLR9 deficiency alleviates doxorubicin-induced cardiotoxicity via the regulation of autophagy," Journal of Cellular and Molecular Medicine, vol. 24, no. 18, pp. 10913-10923, 2020.

[35] L. Zhu, J. Wang, W. Kong et al., "LSD1 inhibition suppresses the growth of clear cell renal cell carcinoma_via_ upregulating P21 signaling," Acta Pharmaceutica Sinica B, vol. 9, no. 2, pp. 324-334, 2019.

[36] C. Hu, X. Zhang, N. Zhang et al., "Osteocrin attenuates inflammation, oxidative stress, apoptosis, and cardiac dysfunction in doxorubicin-induced cardiotoxicity," Clinical and Translational Medicine, vol. 10, no. 3, p. e124, 2020.

[37] M. Wang, C. Mao, L. Ouyang et al., "Long noncoding RNA LINC00336 inhibits ferroptosis in lung cancer by functioning as a competing endogenous RNA," Cell Death and Differentiation, vol. 26, no. 11, pp. 2329-2343, 2019.

[38] G. Tuguzbaeva, E. Yue, X. Chen et al., "PEP06 polypeptide 30 is a novel cluster-dissociating agent inhibiting $\alpha \mathrm{v}$ integrin/ FAK/Src signaling in oral squamous cell carcinoma cells," Acta Pharmaceutica Sinica B, vol. 9, no. 6, pp. 1163-1173, 2019.

[39] X. Zhao, F. Zheng, Y. Li et al., "BPTF promotes hepatocellular carcinoma growth by modulating hTERT signaling and cancer stem cell traits," Redox Biology, vol. 20, pp. 427-441, 2019.

[40] X. Zhang, C. Hu, N. Zhang et al., "Matrine attenuates pathological cardiac fibrosis via RPS5/p38 in mice," Acta Pharmacologica Sinica, vol. 42, no. 4, pp. 573-584, 2021.

[41] W. Zhan, X. Liao, Z. Chen et al., "LINC00858 promotes colorectal cancer by sponging miR-4766-5p to regulate PAK2," Cell Biology and Toxicology, vol. 36, no. 4, pp. 333-347, 2020.

[42] F. Ma, M. G. Ding, Y. Y. Lei et al., "SKIL facilitates tumorigenesis and immune escape of NSCLC via upregulating TAZ/ autophagy axis," Cell Death \& Disease, vol. 11, no. 12, p. $1028,2020$. 
[43] Y. Wu, M. H. Yuan, H. T. Wu et al., "MicroRNA-488 inhibits proliferation and motility of tumor cells via downregulating FSCN1, modulated by Notch3 in breast carcinomas," Cell Death \& Disease, vol. 11, no. 10, p. 912, 2020.

[44] M. Li, H. Xu, and J. Wang, "Optimized functional and structural design of dual-target LMRAP, a bifunctional fusion protein with a 25-amino-acid antitumor peptide and $\mathrm{GnRH}$ fc fragment," Acta Pharmaceutica Sinica B, vol. 10, no. 2, pp. 262-275, 2020.

[45] Y. Jiang, Y. Liang, L. Li et al., "Targeting neddylation inhibits intravascular survival and extravasation of cancer cells to prevent lung-cancer metastasis," Cell Biology and Toxicology, vol. 35, no. 3, pp. 233-245, 2019.

[46] M. J. Lopez-Grueso, D. J. Lagal, A. F. Garcia-Jimenez et al., "Knockout of PRDX6 induces mitochondrial dysfunction and cell cycle arrest at G2/M in HepG2 hepatocarcinoma cells," Redox Biology, vol. 37, article 101737, 2020.

[47] X. Zhang, J. X. Zhu, Z. G. Ma et al., "Rosmarinic acid alleviates cardiomyocyte apoptosis via cardiac fibroblast in doxorubicininduced cardiotoxicity," International Journal of Biological Sciences, vol. 15, no. 3, pp. 556-567, 2019.

[48] F. Y. Liu, D. Fan, Z. Yang et al., "TLR9 is essential for HMGB1mediated post-myocardial infarction tissue repair through affecting apoptosis, cardiac healing, and angiogenesis," Cell Death \& Disease, vol. 10, no. 7, p. 480, 2019.

[49] X. Zhang, C. Hu, X. P. Yuan et al., "Osteocrin, a novel myokine, prevents diabetic cardiomyopathy via restoring proteasomal activity," Cell Death \& Disease, vol. 12, no. 7, p. 624, 2021.

[50] F. Zhou, J. Mei, X. Han et al., "Kinsenoside attenuates osteoarthritis by repolarizing macrophages through inactivating NF-

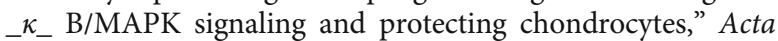
Pharmaceutica Sinica B, vol. 9, no. 5, pp. 973-985, 2019.

[51] C. Hu, X. Zhang, P. Song et al., "Meteorin-like protein attenuates doxorubicin-induced cardiotoxicity via activating cAMP/ PKA/SIRT1 pathway," Redox Biology, vol. 37, article 101747, 2020.

[52] Y. Li, Q. He, X. Wen et al., "EZH2-DNMT1-mediated epigenetic silencing of miR-142-3p promotes metastasis through targeting ZEB2 in nasopharyngeal carcinoma," Cell Death and Differentiation, vol. 26, no. 6, pp. 1089-1106, 2019.

[53] W. Pan, L. Wang, X. F. Zhang et al., "Hypoxia-induced microRNA-191 contributes to hepatic ischemia/reperfusion injury through the ZONAB/cyclin D1 axis," Cell Death and Differentiation, vol. 26, no. 2, pp. 291-305, 2019.

[54] X. Han, H. Jiang, J. Qi et al., "Novel lncRNA UPLA1 mediates tumorigenesis and prognosis in lung adenocarcinoma," Cell Death \& Disease, vol. 11, no. 11, p. 999, 2020.

[55] S. Chen, Y. Li, S. Zhi et al., "WTAP promotes osteosarcoma tumorigenesis by repressing HMBOX1 expression in an m A-dependent manner," Cell Death \& Disease, vol. 11, no. 8, p. 659, 2020.

[56] T. Ren, B. Zheng, Y. Huang et al., "Osteosarcoma cell intrinsic PD-L2 signals promote invasion and metastasis via the RhoAROCK-LIMK2 and autophagy pathways," Cell Death \& Disease, vol. 10, no. 4, p. 261, 2019.

[57] R. Benedetti, C. Papulino, G. Sgueglia et al., "Regulatory interplay between miR-181a-5p and estrogen receptor signaling cascade in breast cancer," Cancers, vol. 13, no. 3, p. 543, 2021.
[58] Y. Wang, Y. X. Fang, B. Dong et al., "Discovery of extracellular vesicles derived miR-181a-5p in patient's serum as an indicator for bone-metastatic prostate cancer," Theranostics, vol. 11, no. 2, pp. 878-892, 2021.

[59] S. Zhang, H. Wan, and X. Zhang, "LncRNA LHFPL3-AS1 contributes to tumorigenesis of melanoma stem cells via the miR-181a-5p/BCL2 pathway," Cell Death \& Disease, vol. 11, no. 11, p. 950, 2020.

[60] L. Li, D. Ye, L. Liu et al., "Long noncoding RNA SNHG7 accelerates proliferation, migration and invasion of nonsmall cell lung cancer cells by suppressing miR-181a-5p through AKT/mTOR signaling pathway," Cancer Management and Research, vol. 12, pp. 8303-8312, 2020.

[61] S. Mutlu, H. Mutlu, S. Kirkbes et al., "The expression of miR181a-5p and miR-371b-5p in chondrosarcoma," European Review for Medical and Pharmacological Sciences, vol. 19, no. 13, pp. 2384-2388, 2015.

[62] J. Xiong and T. Li, "Exploring the association mechanism between metastatic osteosarcoma and non-metastatic osteosarcoma based on dysfunctionality module," Journal of BUON, vol. 25, no. 3, pp. 1569-1578, 2020.

[63] X. Zhang, C. Hu, Y. P. Yuan et al., "Endothelial ERG alleviates cardiac fibrosis via blocking endothelin-1-dependent paracrine mechanism," Cell Biology and Toxicology, vol. 37, no. 6, pp. 873-890, 2021.

[64] X. Zhang, C. Hu, C. Y. Kong et al., "FNDC5 alleviates oxidative stress and cardiomyocyte apoptosis in doxorubicin-induced cardiotoxicity via activating AKT," Cell Death and Differentiation, vol. 27, no. 2, pp. 540-555, 2020.

[65] Q. Wang, M. J. Liu, J. Bu et al., "miR-485-3p regulated by MALAT1 inhibits osteosarcoma glycolysis and metastasis by directly suppressing c-MET and AKT3/mTOR signalling," Life Sciences, vol. 268, article 118925, 2021.

[66] X. Xiao, W. Wang, Y. Li et al., "HSP90AA1-mediated autophagy promotes drug resistance in osteosarcoma," Journal of Experimental \& Clinical Cancer Research, vol. 37, no. 1, p. 201, 2018.

[67] X. Zhang, P. Qu, H. Zhao, T. Zhao, and N. Cao, “COX-2 promotes epithelial-mesenchymal transition and migration in osteosarcoma MG-63 cells via PI3K/AKT/NF- $\kappa \mathrm{B}$ signaling," Molecular Medicine Reports, vol. 20, no. 4, pp. 3811-3819, 2019. 